\title{
The role of GRK6 in animal models of Parkinson's Disease and L-DOPA
}

SUBJECT AREAS:

BEHAVIOUR

RECEPTORS

MODELLING

PATHOLOGY

Received

9 November 2011

Accepted

14 February 2012

Published

5 March 2012

Correspondence and requests for materials should be addressed to R.R.G. (raul. gainetdinov@iit.it)

\section{treatment}

\author{
Francesca Managò', Stefano Espinoza' ', Ali Salahpour², Tatyana D. Sotnikova' ', Marc G. Caron ${ }^{3}$, \\ Richard T. Premont ${ }^{4}$ \& Raul R. Gainetdinov ${ }^{1,3}$
}

'Department of Neuroscience and Brain Technologies, Istituto Italiano di Tecnologia, Genoa, Italy, ${ }^{2}$ Department of Pharmacology and Toxicology, University of Toronto, Toronto (ON), Canada, ${ }^{3}$ Department of Cell Biology, Duke University, Durham (NC), USA, ${ }^{4}$ Department of Medicine, Duke University, Durham (NC), USA.

G protein-coupled Receptor Kinase 6 (GRK6) belongs to a family of kinases that phosphorylate GPCRs. GRK6 levels were found to be altered in Parkinson's Disease (PD) and $\mathrm{D}_{2}$ dopamine receptors are supersensitive in mice lacking GRK6 (GRK6-KO mice). To understand how GRK6 modulates the behavioral manifestations of dopamine deficiency and responses to L-DOPA, we used three approaches to model PD in GRK6-KO mice: 1) the cataleptic response to haloperidol; 2) introducing GRK6 mutation to an acute model of absolute dopamine deficiency, DDD mice; 3) hemiparkinsonian 6-OHDA model. Furthermore, dopamine-related striatal signaling was analyzed by assessing the phosphorylation of AKT/GSK3 $\beta$ and ERK1/2. GRK6 deficiency reduced cataleptic behavior, potentiated the acute effect of L-DOPA in DDD mice, reduced rotational behavior in hemi-parkinsonian mice, and reduced abnormal involuntary movements induced by chronic L-DOPA. These data indicate that approaches to regulate GRK6 activity could be useful in modulating both therapeutic and side-effects of L-DOPA.

P

arkinson's disease (PD) is a neurodegenerative disorder characterized by the loss of nigrostriatal dopaminergic neurons, which results in the motor deficits typical of this disease ${ }^{1,2}$. The most widely used pharmacotherapy is L-DOPA, which markedly improves motor deficits; however, long-term therapy often results in the development of motor side effects such as dyskinesia ${ }^{3-5}$.

The physiological functions of dopamine are mediated through the activation of dopamine receptors that belong to the family of G protein-coupled receptors (GPCRs), which can be divided into two major categories: $\mathrm{D}_{1^{-}}$ like $\left(D_{1}, D_{5}\right)$ and $D_{2}$-like $\left(D_{2}, D_{3}, D_{4}\right)$ receptors ${ }^{6,7}$. The activation of a GPCR initiates the classical G proteindependent signaling cascade, but also sets in place series of molecular interactions that induce feedback regulation of $G$ protein coupling, endocytosis of receptor, and signaling through $G$ protein-independent signal transduction pathways involving $\beta$ arrestin proteins ${ }^{8}$. In particular, a non-canonical $\beta$ arrestin 2-mediated pathway plays an important role in the behavioral responses to dopamine'. G protein-coupled receptor kinases (GRKs) promote ßarrestin recruitment by phosphorylating activated GPCRs, thereby playing a pivotal role in these subsequent desensitization and/or signaling events ${ }^{10}$. GRK6 is the most abundant isoform in the caudate/putamen, and postsynaptic $\mathrm{D}_{2} / \mathrm{D}_{3}$ dopamine receptors are the physiological targets of this kinase ${ }^{11,12}$. Both in vitro and in vivo experiments have demonstrated that GRK6 is involved in the desensitization of $\mathrm{D}_{2} / \mathrm{D}_{3}$ dopamine receptors, resulting in altered ability of $\mathrm{D}_{2}$-like, but not $\mathrm{D}_{1}$-like dopamine receptor agonists to affect locomotor behaviors in GRK6-KO mice ${ }^{12}$. Based on these observations, it has been suggested that a pharmacological strategy targeted on GRK6 expression or activity may be beneficial in conditions when dopaminergic signaling is limited, such as Parkinson's disease ${ }^{12}$. In a study involving MPTP-treated monkeys as a model of PD, an elevated expression level of GRK6 was found in the caudate/putamen and ventral striatum, which could be normalized by L-DOPA treatment ${ }^{13}$. On the other hand, 6-OHDA-lesioned rats showed a reduction of GRK6 levels in the caudal caudate/putamen and globus pallidus, while GRK6 expression was up-regulated in the rostral caudate/putamen and nucleus accumbens of these animals ${ }^{14}$. Furthermore, post mortem analysis of the striatum of PD patients without dementia that were treated with L-DOPA for several years showed a decreased concentration of GRK6, in agreement with work on MPTP-treated monkeys ${ }^{13}$. It is also possible that altered levels of GRK6 can result in dysregulation of DA receptors, inducing an abnormal signaling, thus contributing to the core motor deficits observed in PD. 
A recent study has convincingly demonstrated that over-expression or reduction of the level of GRK6 expression in the striatum has significant modulatory effect on the antiparkinsonian and sideeffects of L-DOPA ${ }^{15}$. Furthermore, over-expression of GRK6 in rats promotes the internalization not only of $D_{2} R$, but also of $D_{1} R$, thus normalizing the signaling through the regulation of the $\mathrm{D} 1$ receptor as well ${ }^{15}$. Exaggerated signaling of striatal $D_{1} R^{16-18}, D_{2} R^{19}$ and $D_{3} R^{20-}$ ${ }^{22}$ following chronic L-DOPA has been implicated in L-DOPAinduced dyskinesia (LID) in rodents and primates, suggesting that normalization of this excessive signal may be beneficial for preventing the development of LIDs. Indeed, over-expression of GRK6 alleviated LIDs in both rat and monkey models of $\mathrm{PD}^{15}$, likely through reducing the abnormal signaling of dopamine receptors related to LID. Taken together, these studies indicate that the changes in GRK6 may play an important role in the pathogenesis of PD and in the responses to anti-PD drugs. For this reason, it is important to investigate in detail the mechanisms of GRK6 involvement in modulating the expression of PD symptoms, as well as in responses to L-DOPA therapy. Therefore, we assessed how behavioral and cellular responses are altered in mouse models of PD that are also deficient in GRK6 expression. Moreover, these GRK6-deficient mouse models of $\mathrm{PD}$ provided an opportunity to investigate responses to acute and chronic treatment with L-DOPA in order to understand how reduced GRK6 levels affect the therapeutic and side-effects of LDOPA.

\section{Results}

Reduced expression of haloperidol-induced catalepsy in GRK6KO mice. To evaluate the impact of GRK6 deficiency on expression of haloperidol-induced catalepsy, a physiological output of postsynaptic $\mathrm{D}_{2}$ dopamine receptor blockade ${ }^{23}$, we administered three doses of haloperidol, 0.5 (Fig. 1A), 1 (Fig. 1B) and 2 (Fig. 1C) $\mathrm{mg} / \mathrm{kg}$, i.p., and tested animals in the bar catalepsy test every hour for four hours after treatment. The results of these measurements were subjected to a Two-way ANOVA analysis, and when there was an effect of all interactions between factors $(p \leq 0.05)$, the significance was further analyzed by Bonferroni post-hoc. As presented in these graphs, WT and GRK6-KO mice injected with $0.5,1$ or $2 \mathrm{mg} / \mathrm{kg}$ of haloperidol, but not saline, demonstrated a dose-dependent increase of catalepsy that varied over time. However, the expression and dynamics of haloperidol-induced catalepsy was altered in GRK6KO mice. GRK6-KO mice injected with the $0.5 \mathrm{mg} / \mathrm{kg}$ haloperidol (Fig. 1A) had cataleptic behavior similar to WT mice during the first two hours, but a reduction of this behavior was observed at the third and fourth hour. Two-way ANOVA for repeated measures revealed an effect of all three factors interaction (Category of Time $\mathrm{x}$ Treatment $x$ Genotype, $\mathrm{F}_{9,139}=4.76$ ) and, in particular, Bonferroni analysis has shown a significant difference between WT and GRK6$\mathrm{KO}$ at the fourth hour $(\mathrm{p}<0.05)$ in the same group (Haloperidol Treatment). GRK6-KO mice administered with $1 \mathrm{mg} / \mathrm{kg}$ of haloperidol showed a different pattern of development of catalepsy. These mice demonstrated an increase in cataleptic behavior along the same time frame as WT group, however, as presented in Fig. 1B, their response is reduced significantly compared to WT mice (main effect of the interaction of Time $\mathrm{x}$ Genotype $\mathrm{x}$ Treatment, $\left.\mathrm{F}_{9,183}=2.71 ; \mathrm{p}<0.05\right)$. With the highest dose of haloperidol tested $(2 \mathrm{mg} / \mathrm{kg}$, i.p., Fig. $1 \mathrm{C})$ we also observed an increase of the cataleptic behavior of the GRK6-KO mice along the time, however their total response was significantly lower than in WT mice (main effect of interaction factors Time $\mathrm{x}$ Genotype $\mathrm{x}$ Treatment $\left.\mathrm{F}_{9,171}=5.16, \mathrm{p}<0.0001\right)$.

pAkt and pGSK3 $\beta$ levels in response to haloperidol in GRK6-KO mice. It has been demonstrated that activation of $\mathrm{D}_{2}$-class dopamine receptors in the striatum can promote $\beta$ arrestin 2-mediated Akt/ GSK3 $\beta$ signaling ${ }^{24}$. To clarify the contribution of GRK6-mediated
Haloperidol $0.5 \mathrm{mg} / \mathrm{kg}$

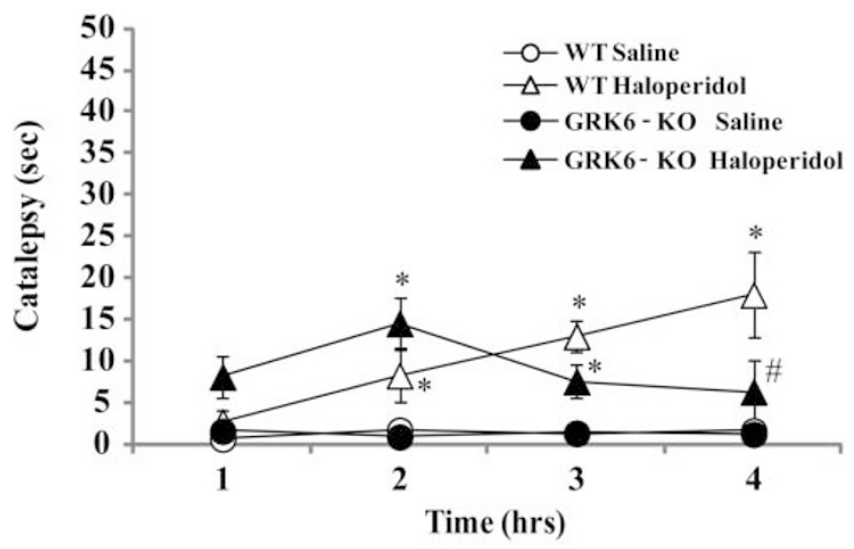

B

Haloperidol $1 \mathrm{mg} / \mathrm{kg}$

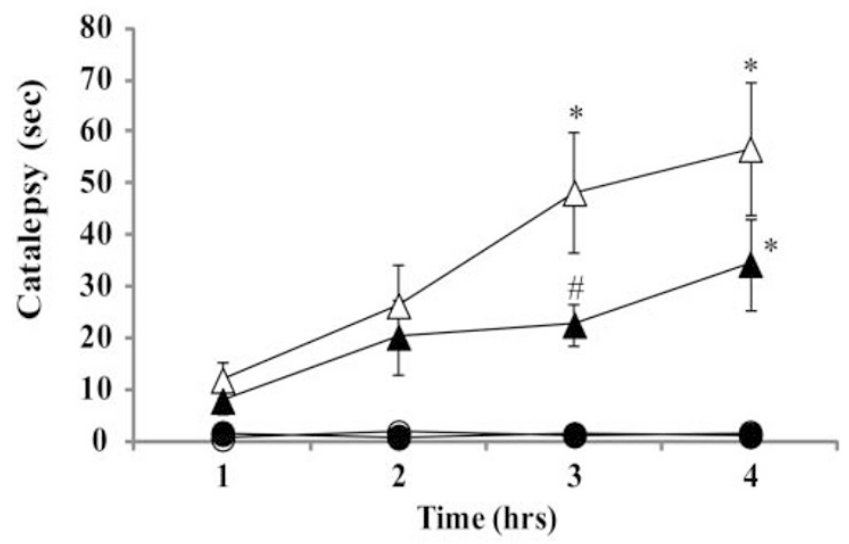

C

Haloperidol $2 \mathrm{mg} / \mathrm{kg}$

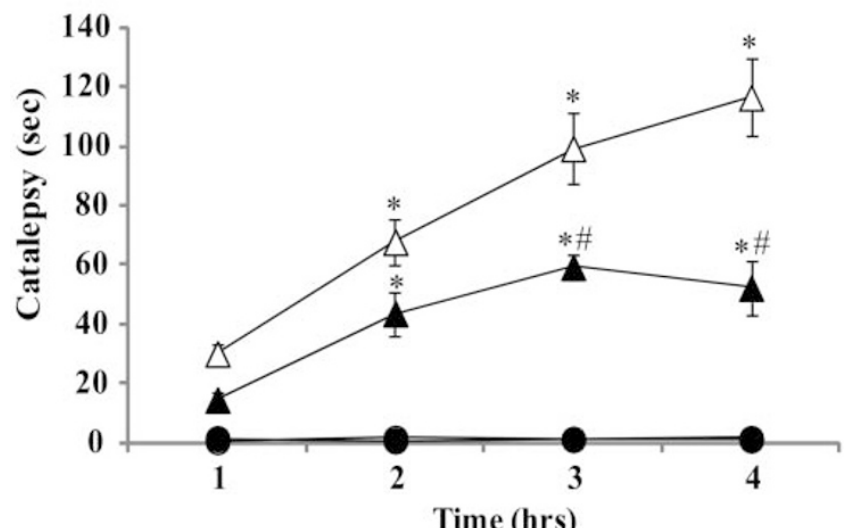

Figure 1 The dynamics of haloperidol-induced catalepsy in GRK6 deficient mice. The histograms illustrate the time the animals spent to remove both front paws from the bar (as index of cataleptic behavior). All the animals were tested at 1, 2, 3 and 4 hours (T1, T2, T3 and T4) after the drug injection. 1A. Effect of haloperidol $0.5 \mathrm{mg} / \mathrm{kg}$, i.p. 4 groups: WT Saline $(n=12)$ and Haloperidol $(n=7)$; GRK6-KO Saline $(n=13)$ and Haloperidol $(\mathrm{n}=7)$. 1B. Effect of haloperidol $1 \mathrm{mg} / \mathrm{kg}$, i.p. 4 groups: WT Saline $(\mathrm{n}=12)$ and Haloperidol $(\mathrm{n}=19)$; GRK6-KO Saline $(\mathrm{n}=13)$ and Haloperidol

$(n=21) .1$. Effect of haloperidol $2 \mathrm{mg} / \mathrm{kg}$, i.p. 4 groups: WT saline $(\mathrm{n}=12)$ and Haloperidol $(n=17)$; GRK6-KO saline $(n=13)$ and Haloperidol $(n=19)$. ${ }^{*} \mathrm{p}<0.05$ vs Saline in the same group of genotype; \# $\mathrm{p}<0.05$ vs WT in the same group of dose (Bonferroni post-hoc). 
regulation of $\mathrm{D}_{2}$ dopamine receptors to this pathway, we performed analysis of the levels of pAkt (Thr308) and pGSK3 $\beta$ (Ser9) in the striatum of drug-naive WT and GRK6-KO mice. These experiments revealed that under basal conditions, GRK6-KO mice show only a trend toward increased pAkt levels, but have significantly increased levels of pGSK3 $\beta$ compared to WT mice (Fig. 2A-C). Next, we analyzed the phosphorylation levels of these two kinases following 1 or $2 \mathrm{mg} / \mathrm{kg}$ doses of haloperidol, 5 hours after administration, as these conditions demonstrated the strongest response in the catalepsy test. In agreement with previous results ${ }^{25}$, the phosphorylation of Akt in WT increases as a result of $\mathrm{D}_{2} \mathrm{R}$ antagonist action in a dose-dependent pattern (Fig. $2 \mathrm{D}$ and $2 \mathrm{E}$ ) (One-way ANOVA, $\mathrm{F}_{2,17}=9,658, \mathrm{p}=0,0016$ ). Tukey HSD post hoc test revealed a significant difference between the haloperidol groups versus the saline control. We noted a similar significant increase of phosphorylation level for GSK3 $\beta$ in WT mice (Fig. 2D and 2F) (Oneway ANOVA, $\left.\mathrm{F}_{2,17}=3,951, \mathrm{p}=0,03\right)$. In contrast, in GRK6-KO mice, pAkt levels were not significantly altered following 1 or $2 \mathrm{mg} / \mathrm{kg}$ haloperidol, compared to the vehicle-treated control (Fig. 2G and $2 \mathrm{H})$. In a similar way, the phosphorylation of GSK3 $\beta$ was only modestly changed in GRK6-KO mice following haloperidol treatment, with significantly increased level of pGSK3 $\beta$ following 1 but not $2 \mathrm{mg} / \mathrm{kg}$ of the drug (Fig. 2G and 2I) (One-way ANOVA, $\left.\mathrm{F}_{2,16}=7,822, \mathrm{p}=0,004\right)$.

Basal locomotor activity of DAT-KO and DAT/GRK6 double KO mice. To study the role of GRK6 under conditions of severe acute DA deficiency, we developed GRK6-deficient DDD mice ${ }^{26}$. For this purpose, we first generated DAT/GRK6 double KO mice by crossing heterozygous mice for dopamine transporter (DAT) and GRK6, and then crossing double heterozygous mice for DAT/ GRK6 to obtain double DAT/GRK6 KO mice. Double DAT/GRK6 $\mathrm{KO}$ mice are viable and do not demonstrate any obvious alterations beyond those observed in single knockout DAT-KO or GRK6-KO mice $^{12,27,28}$. We compared untreated DAT-KO and DAT/GRK6 double $\mathrm{KO}$ mice for the potential alterations related to GRK6
A

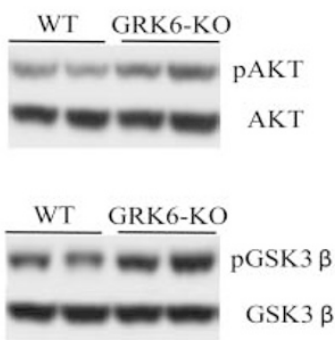

D

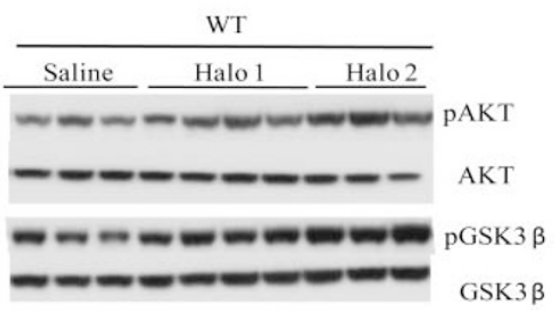

G

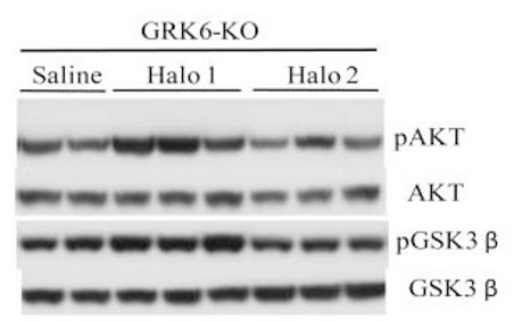

B

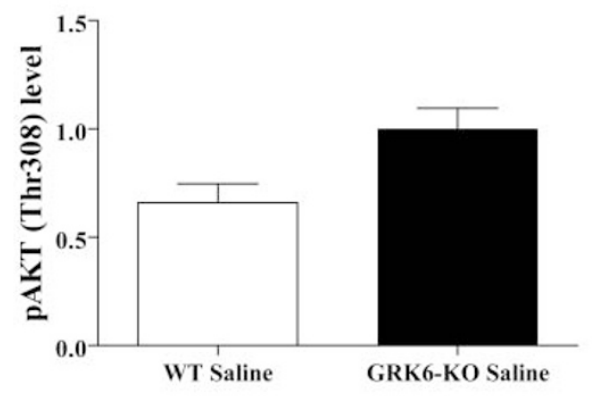

E

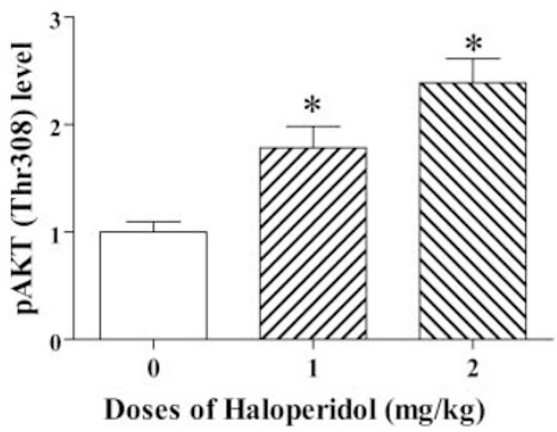

H GRK6-KO

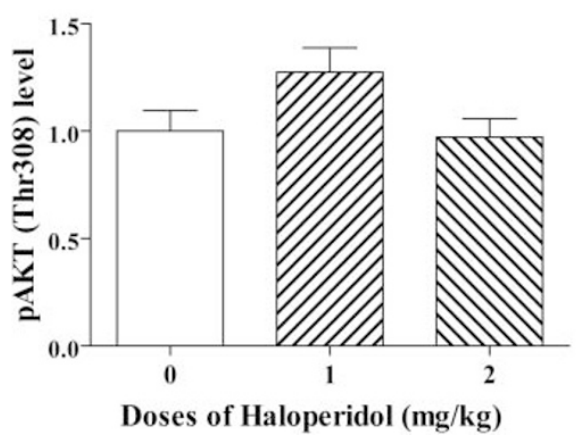

C

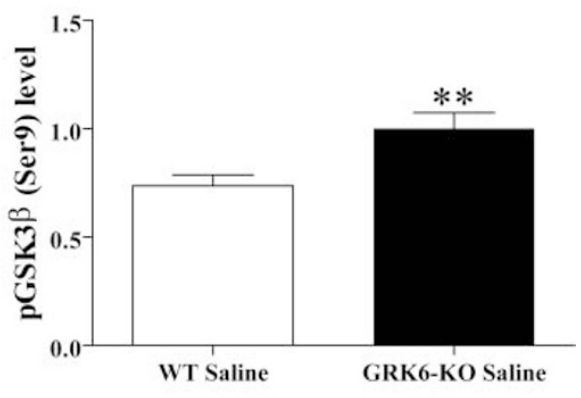

WT

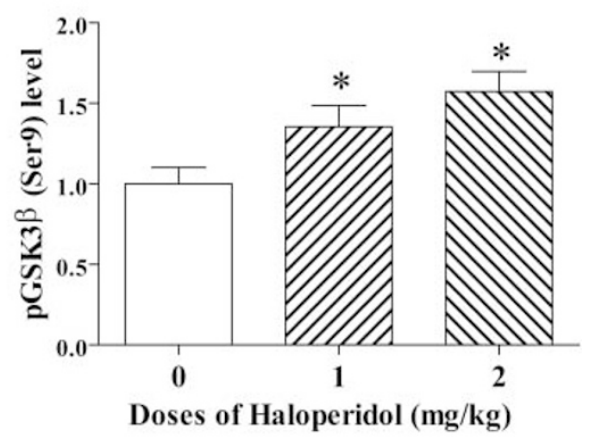

GRK6-KO

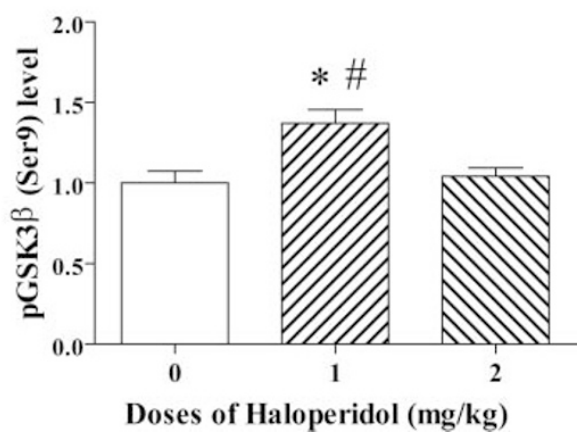

Figure $2 \mid$ Levels of pAKT and pGSK3 $\beta$ in the striatum of WT and GRK6-KO mice following haloperidol. Western blots and densitometric analysis of relative levels of pAKT (Thr-308) or pGSK3 $\beta$ (Ser9) were examined in extracts prepared from the striatum of WT and GRK6-KO untreated mice (2A-C), and WT (2D-F) or GRK6-KO (2G-I) treated mice ( $\mathrm{n}=7-19$ per group) with saline and two doses of haloperidol ( 1 and $2 \mathrm{mg} / \mathrm{kg}$, i.p.). One-Way Anova analysis with Tukey HSD post hoc test. Two-tailed Mann Whitney test. In all experiments total kinase levels in extracts were used as loading controls for measurement of phospho-protein levels. Data are means $\pm \mathrm{SEM} ;{ }^{*} \mathrm{p}<0.05,{ }^{*} \mathrm{p}<0.01$ vs respective controls; \#p $<0.05 \mathrm{vs} 2 \mathrm{mg} / \mathrm{kg}$ group. 
deficiency at the level of basal locomotor activity. Double KO mice demonstrated significantly increased level of total distance traveled in comparison to spontaneously hyperactive DAT-KO mice, and this difference was attributable mainly to diminished habituation at later times, indicating that combination of high DA extracellular levels due to DAT deficiency with uncontrolled DA receptor supersensitivity caused by the lack of GRK6 leads to more pronounced hyperactivity ${ }^{12}$ (Fig. 3). The analyses of the variance for repeated measures have shown an effect of Time $x$ Genotype interaction factors $\left(\mathrm{F}_{23,483}=3.168, \mathrm{p}<0.0001\right)$. Notably, activity levels of GRK6-KO mice are not different from their wild type controls (Ref 12).

Effect of L-DOPA in DDD mice. To evaluate the acute effects of LDOPA in dopamine deficiency model, we first administered amethyl-p-tyrosine ( $\alpha \mathrm{MT} ; 250 \mathrm{mg} / \mathrm{kg}$, i.p.), a potent irreversible inhibitor of TH, to DAT-KO and DAT/GRK6 double KO mice to deplete dopamine in the brain of these animals (dopamine-deficient DAT-KO mice, DDD mice ${ }^{26}$ ) (Fig. 4). It is known that $\alpha$ MT induces virtual disappearance of extracellular DA in DDD mice while LDOPA effectively restores DA levels in this model as evidenced by in vivo microdialysis experiments ${ }^{26,29}$. One hour after $\alpha \mathrm{MT}$, the dopamine-deficient animals received an injection of L-DOPA/ Carbidopa $(10 / 10 \mathrm{mg} / \mathrm{kg}$, i.p.), followed by another dose of LDOPA (20 mg/kg, i.p.) one hour later (Fig. 4A). In a second group of animals, one hour after $\alpha \mathrm{MT}$ injection, DDD mice received an injection of L-DOPA/Carbidopa at higher dose $30 / 10 \mathrm{mg} / \mathrm{kg}$, i.p. (Fig. 4B). After $\alpha \mathrm{MT}$ treatment, all the mutant mice became akinetic due to dopamine deficiency, as reported before ${ }^{26}$. As expected, L-DOPA treatment reversed this phenotype and induced an increased activity proportionally to the dose of the drug (Fig. 4AB). However, the effect of L-DOPA on the double KO was much stronger in comparison to the DAT-KO mice. The analyses of the variance on the total distance have shown a significant effect for the interaction factors Time $\mathrm{x}$ Genotypes in both experiments (first group of treatment (Fig. 4A) $\mathrm{F}_{36,504}=4.928, \mathrm{p}<0.0001$; second group of treatment (Fig. $4 \mathrm{~B})\left(\mathrm{F}_{36,540}=2, \mathrm{p}=0.0006\right)$.

Effect of GRK6 deficiency in unilateral 6-OHDA model of PD. Lesion verification: quantitative western blotting for $\mathrm{TH}$ of the striatum. To develop unilateral 6-OHDA model in normal and mutant mice we elected to use intrastriatal injection of the toxin, which has been shown to develop significant lesion in the dopamine terminal regions with lesser mortality and larger therapeutic window for L-DOPA treatment in comparison to

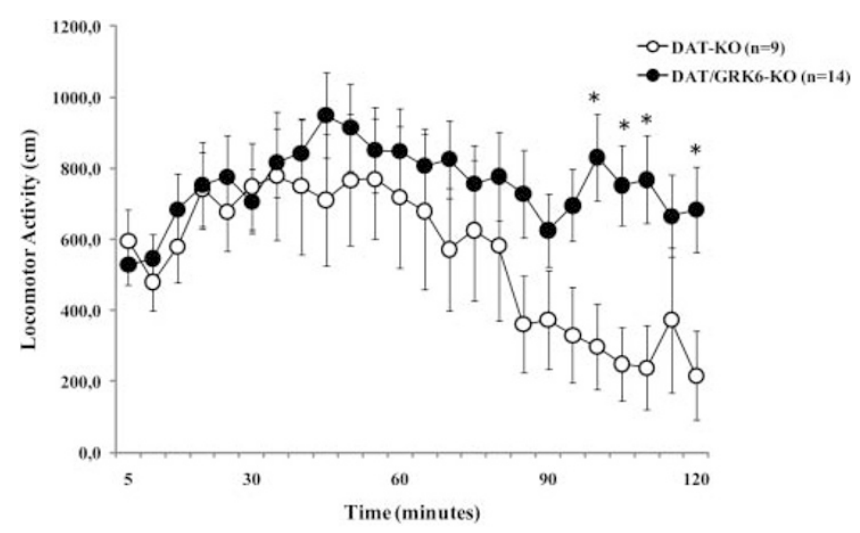

Figure 3 | Basal locomotor activity of GRK6/DAT double KO mice. Spontaneous locomotor activity of DAT-KO and GRK6/DAT-KO mice was measured through 2 hours recording. One-Way Anova for repeated measures, Tukey HSD post hoc analysis. ${ }^{*} \mathrm{p}<0.05$ vs DAT-KO in the same group of time point.
A

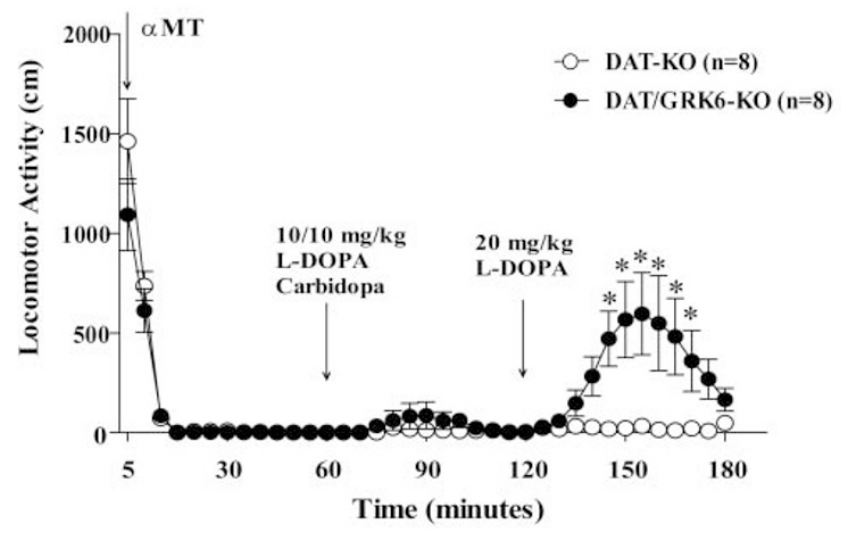

B

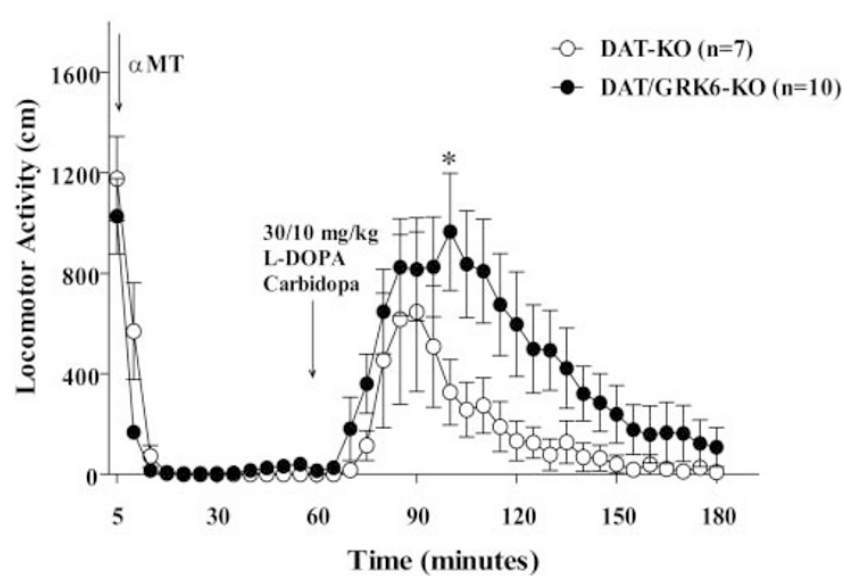

Figure $4 \mid$ Effect of L-DOPA on locomotor activity of GRK6-deficient DDD mice. After 30 minutes of habituation, all groups received $\alpha \mathrm{MT}$ $250 \mathrm{mg} / \mathrm{kg}$, i.p. and 1 hour later they received an injection of L-DOPA/ Carbidopa 10/10 mg/kg, i.p. followed by of L-DOPA $20 \mathrm{mg} / \mathrm{kg}$, i.p. one hour later (4A). A second group of animal was treated with $30 / 10 \mathrm{mg} / \mathrm{kg}$, i.p. of L-DOPA/Carbidopa (4B). Statistical analysis: One-Way Anova for repeated measures, Bonferroni post hoc analysis. ${ }^{*} \mathrm{p}<0.05$ vs DDD mice at the same time point.

medial forebrain bundle (MFB) lesion protocol $^{30}$. To verify the degree of lesion induced by intrastriatal 6-OHDA infusion, we have analyzed the TH immunoreactivity of the striatal samples by western blot in operated mutant and WT mice. We used as control the mean of $\mathrm{TH}$ levels in the striatum of sham-operated mice separately for WT and GRK6-KO; then we compared each band of the lesioned mice with the appropriate control (representative blots are shown in Supplementary Fig. S1). Only the data from WT or GRK6-KO mice that demonstrated TH depletion of more than $75 \%$ compared to their control were included in the statistical analysis $^{30}$.

Rotational behavior and AIMs. Following chronic treatment with LDOPA/carbidopa, unilaterally-lesioned WT and GRK6-KO animals showed progressive increase in rotational behaviors known to be induced by this treatment ${ }^{14,15,22}$. In contrast, non-lesioned WT and GRK6-KO mice (sham-operated) did not demonstrate turning behavior. However, unexpectedly, we found a reduced rotational response to L-DOPA in GRK6-KO-lesioned compared to the WT-lesioned mice. Two-way ANOVA has shown an effect of the Days Treatment $x$ Genotype $x$ Lesion interaction $\left(F_{3,90}=3,539, p=0,01\right)$, with Tukey HSD post hoc analysis revealing significant points as shown in 


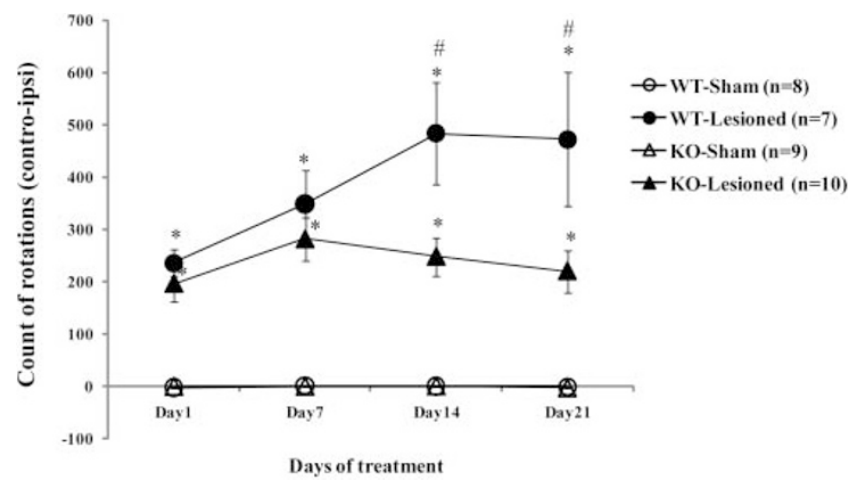

Figure 5 | L-DOPA-induced rotations in 6-OHDA lesioned WT and GRK6-KO. In these experiments we had two groups for WT: Sham and Lesioned and two for GRK6-KO: Sham and Lesioned. All the animals were treated with $20 / 12 \mathrm{mg} / \mathrm{kg}$ of L-DOPA/Carbidopa for 21 days. The rotations (means, \pm SEM) are counted as controlateral minus ispilateral rotations. Two-Way Anova for repeated measures, Tukey HSD post hoc. ${ }^{*} \mathrm{p}<0.05$ vs Sham in the same group of genotype and time point; $\# \mathrm{p}<0.05$ vs GRK6-KO lesioned in the same time point.

the graph (Fig. 5). Furthermore, we analyzed a time course of rotations of the last day of treatment (Supplementary Fig. S2) and found a significant difference in the circling behavior along all the time periods tested between WT and GRK6-KO with an effect of all three factors of interaction $\left(\mathrm{F}_{5,150}=2,917, \mathrm{p}=0.01\right)$. While this finding seems not to be in agreement with the recent publication of Ahmed, et al $(2010)^{15}$, we note that there are significant differences in experimental procedures between the two studies. In particular, we used full knockout mice for GRK6 that have deficiency in GRK6 in both striata, while Ahmed et al (2010) ${ }^{15}$ induced knockdown or overexpression of GRK6 only in the lesioned striatum. In our case, the dopaminergic supersensitive state due to GRK6 deficiency ${ }^{12}$ appears on both sides, thus reducing potential for rotations in lesioned animals, while manipulating GRK6 only on the lesioned side will likely exacerbate the differences between sides, increasing potential for rotations ${ }^{15}$. Furthermore, similarly to rotational experiments, GRK6-KO mice with unilateral lesion showed a reduced frequency of AIMs compared to WT mice (Fig. 6A-C). The AIM rating increased with days of treatment as shown in the graph of the total dyskinesia in a similar way in both groups (combined scores of limb and axial AIMs) (Fig. 6C). However, Two-way ANOVA analysis has revealed an effect of the Genotype factor $\left(\mathrm{F}_{1,15}=12.512, \mathrm{p}=0.003\right)$, indicating that there is a difference in the AIMs manifestations between the genotypes. In particular, GRK6-KO mice showed reduced levels of AIMs compared to the WT controls. We obtained the same statistical result for axial dyskinesia (Fig. $6 \mathrm{~A})\left(\mathrm{F}_{1,15}=15.412\right.$, $\mathrm{p}=0.001$ ). Regarding limb dyskinesia (Fig. 6B), both WT and GRK6KO showed an increase of AIM rating with time but GRK6-KO mice had a somewhat reduced level of dyskinesia compared to WT group despite not reaching statistically significant effect of the factors. Analysis of the time course of AIMs at the last day of treatment (Supplementary Fig. S3) revealed an effect of the Genotype in the total $\left(\mathrm{F}_{1,15}=6.091, \mathrm{p}=0.02\right)$ and axial $\left(\mathrm{F}_{1,15}=5,170, \mathrm{p}=0.03\right)$ AIMs score. In the total AIM scoring, we did not consider the orolingual subtype of $\mathrm{AIMs}^{31}$ because of experimental difficulties in detecting these behaviors in our study.

pERK1/2, pAkt and pGSK3 $\beta$ levels in response chronic L-DOPA treatment. It has been reported that in 6-OHDA-lesioned mice, chronic treatment with L-DOPA results in elevated ERK phosphorylation $^{32}$. While no significant difference in pERK1 or pERK2 level was seen between WT and GRK6-KO mice for either shamoperated or lesioned groups treated chronically with L-DOPA for 21
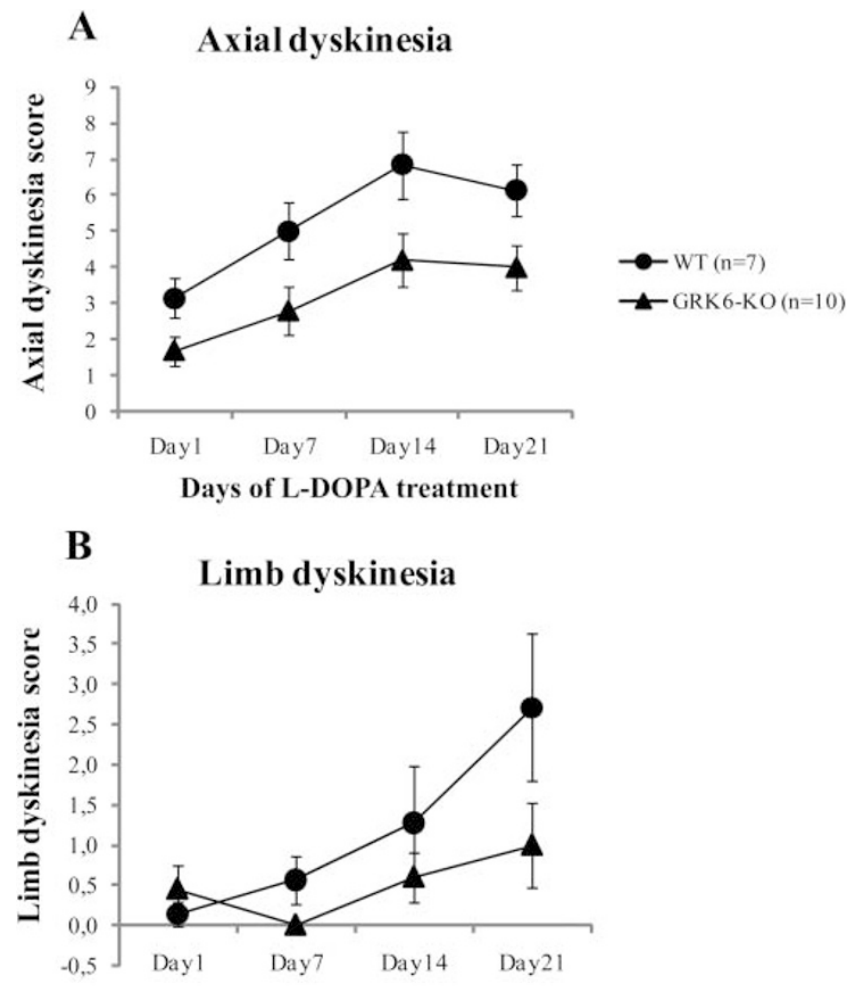

Days of L-DOPA treatment

C

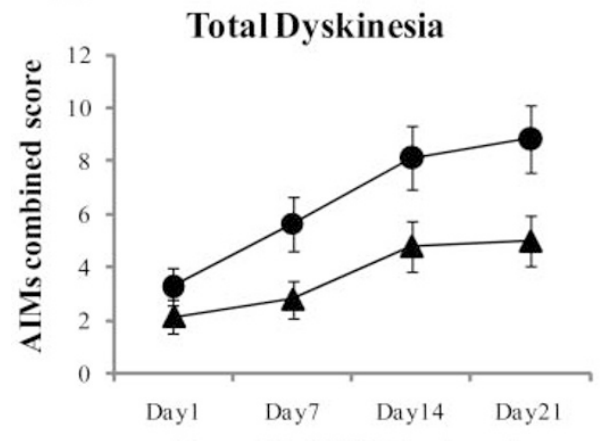

Days of L-DOPA treatment

Figure 6 Chronic L-DOPA induced AIMs in GRK6-KO mice. Axial AIMs (6A); Limb AIMs(6B); Combined AIMs score (6C). WT and GRK6KO 6-OHDA-lesioned animals received L-DOPA treatment for 21 days and AIMs were scored using the rodent AIM rating system described in Cenci and Lundbland $(2007)^{51}$. No significant AIMs were detected in sham-operated controls.

days (Fig. 7A-F), we observed a significant increase of pERK2 in LDOPA-treated WT lesioned mice compared to their sham-operated controls (Fig. 7G and 7I). The same trend of increase can be seen for the pERK1 levels; however, this effect does not reach statistical significance (Fig. $7 \mathrm{G}$ and $7 \mathrm{H}$ ). At the same time, we found no significant differences in phosphorylation of ERK1 or ERK2 between GRK6-KO lesioned and sham-operated mice after chronic L-DOPA treatment (Fig. 7J-L), conditions that did show a reduced level of rotations and AIMs. This result seems to be in agreement with the positive correlation between AIMs rating and pERK1 and pERK2 levels reported by other labs ${ }^{32}$. The total levels of ERK1 and ERK2 were not changed in any of the groups (data not shown). We have also analyzed the levels of phosphorylation of pAkt and pGSK3 $\beta$ proteins in WT and GRK6-KO chronically treated with L-DOPA. However, in these experiments we did not observe any significant differences between any of the group analyzed (data not shown). 
A

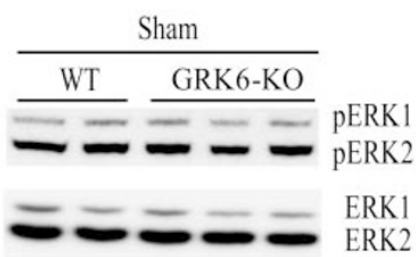

D

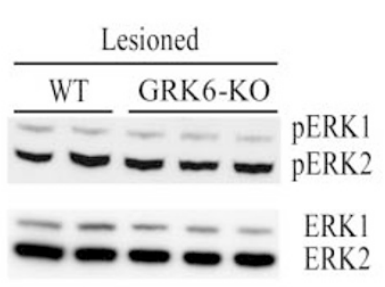

G

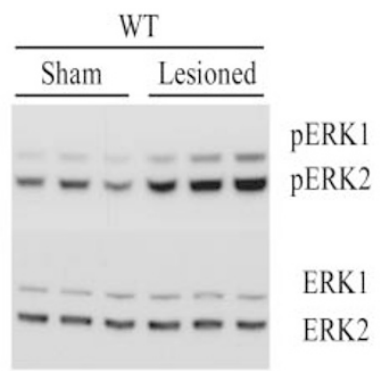

J

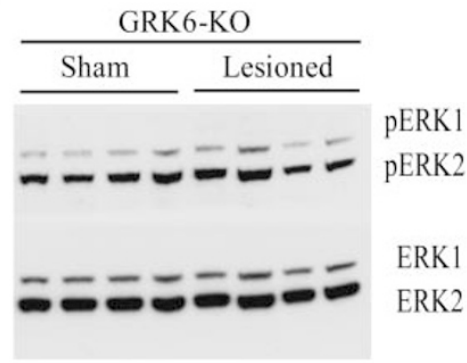

B

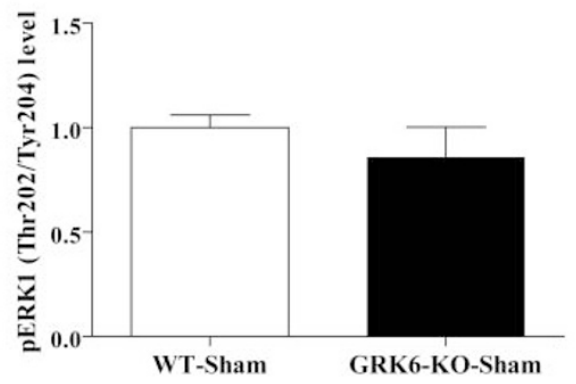

E

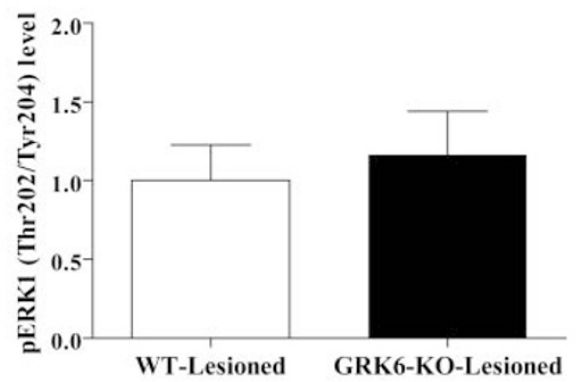

H

WT

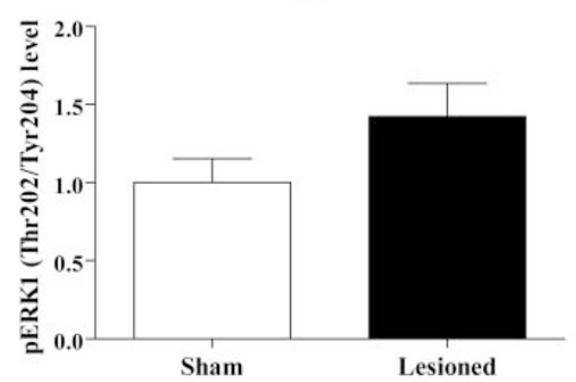

K

GRK6-KO

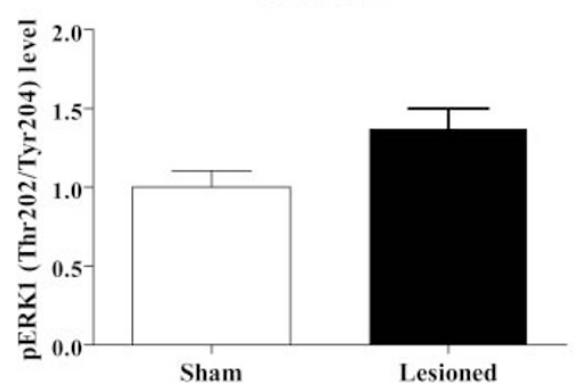

C

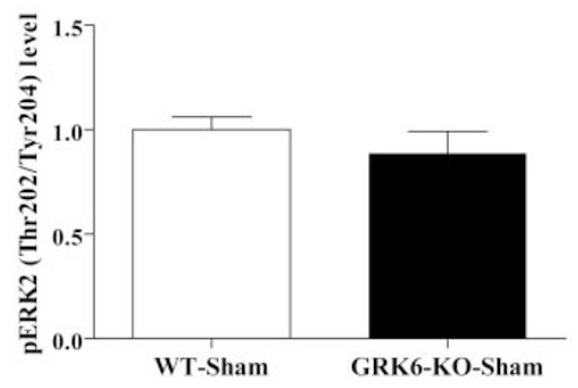

F
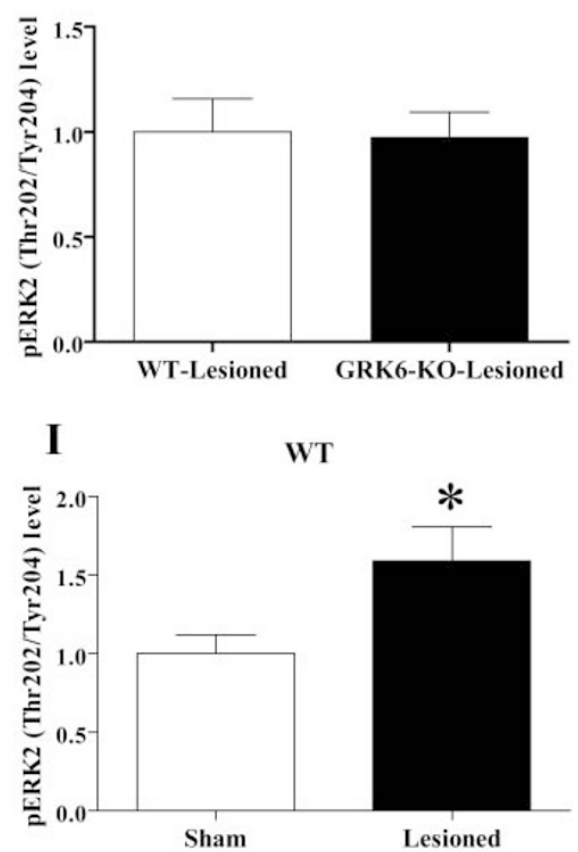

L

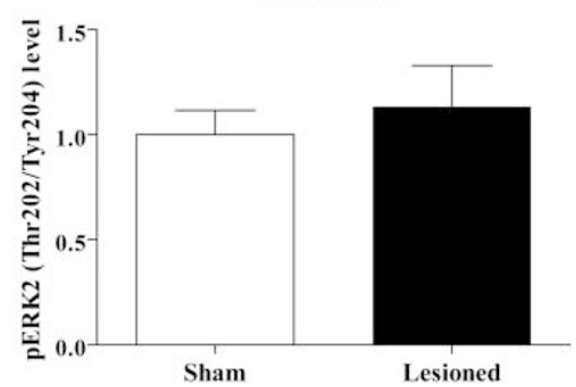

Figure 7 | Levels of pERK1 and pERK2 in the striatum of unilaterally lesioned L-DOPA treated WT and GRK6-KO mice. Western blots and densitometric analysis of relative levels of pERK1 and pERK2 were examined in extracts prepared from the striatum of WT and GRK6-KO chronically treated with L-DOPA for 21 days ( $\mathrm{n}=7-10$ per group). Comparison of pERK1 and pERK2 levels in sham-operated WT and GRK6-KO mice (7A-C) and lesioned WT and GRK6-KO mice (7D-F). Comparison of pERK1 and pERK2 levels in sham-operated and lesioned WT mice (7G-I) and sham-operated and lesioned GRK6-KO mice (7J-L). Total protein levels in extracts were used as loading controls for measurement of phospho-protein levels. Data are means $\pm \mathrm{SEM} ;{ }^{*} \mathrm{p}<0.05$, two-tailed Mann Whitney test.

GRK6 overexpression in vitro modulates both the $G$ proteinmediated cAMP signaling and Barrestin 2 recruitment. To gain more insights on the mechanism of GRK6 involvement in $\mathrm{D}_{2}$ dopamine receptor signaling and regulation we have used an in vitro cell-based assay to test the GRK6 influence on G protein-dependent and independent processes. Thus, we evaluated the effect of overexpression of GRK6 in HEK-293 cells on the ability of $\mathrm{D}_{2} \mathrm{R}$ to modulate cAMP levels and Barrestin 2 recruitment using two different Bioluminescence Resonance Energy Transfer (BRET) techniques that has been proven to have great utility in studying these phenomena ${ }^{33}$. In the first experiment, cells were transfected with a CAMP EPAC biosensor that appears to be sensitive tool in monitoring cAMP fluctuations ${ }^{34}$. Cells were pretreated for 5 minutes with the $\mathrm{D}_{2}$ agonist quinpirole at $10 \mu \mathrm{M}$ and then stimulated with forskolin at $3 \mu \mathrm{M}$. As shown in Fig. 8A, in WT cells quinpirole was able to induce a decrease of about $50 \%$ of forskolin stimulation, but 
when GRK6 was overexpressed this effect was significantly impaired (quinpirole WT $=47 \pm 1.6 \%$, quinpirole GRK6 $=89 \pm 4.6 \%$, $\mathrm{p}<0.05, \mathrm{n}=4)$. These data confirm previous observations revealing that GRK6 overexression reduces the coupling of $G$ protein to $D_{2}$ receptors as measured in $\left[{ }^{35} \mathrm{~S}\right] \mathrm{GTP} \gamma \mathrm{S}$ binding experiments ${ }^{12}$.

Further, we investigated the role of GRK6 in $\beta$ arrestin 2 recruitment to $D_{2}$ receptors. We transfected HEK-293 cells with $D_{2}$-Rluc and $\beta$ arrestin 2-YFP 33 and stimulated the cells with quinpirole at 10 $\mu \mathrm{M}$. As expected, quinpirole produced an increase in BRET ratio in

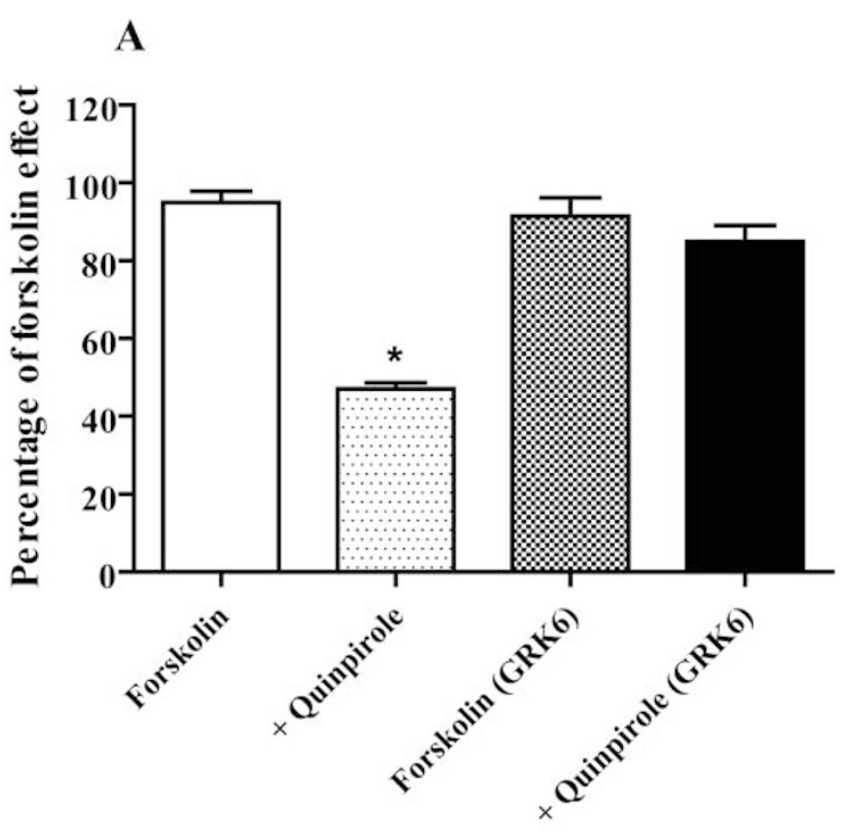

B

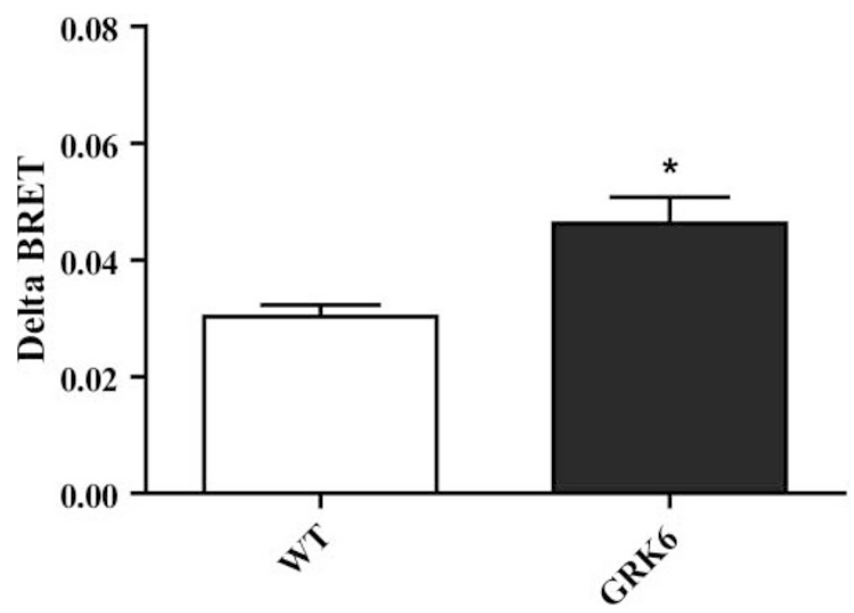

Figure 8 Effect of GRK6 overexpression on the D2R-dependent G protein-mediated signaling and Barrestin 2 recruitment. $8 \mathrm{~A}$, In cells transfected with EPAC sensor and $\mathrm{D}_{2} \mathrm{R}$, cAMP level was monitored for 25 minutes. Cells were pretreated with quinpirole at $10 \mu \mathrm{M}$ and then cAMP production was stimulated by forskolin at $3 \mu \mathrm{M}$. In wild type cells quinpirole decreased forskolin effect by about $50 \%(\mathrm{p}<0.05)$, while in GRK6 overexpressing cells it had no significant effect. $8 \mathrm{~B}$, $\beta$ arrestin 2 recruitment was evaluated by coexpression of $\mathrm{D}_{2}$-Rluc and $\beta$ arrestin 2YFP. Cells were stimulated with quinpirole at $10 \mu \mathrm{M}$ and then BRET ratio was monitored for 25 minutes. Quinpirole was able to induce an increase in BRET ratio. Coexpression of GRK6 produced a significant increase in delta-BRET $(\mathrm{p}<0.05)$. All values are expressed as means \pm SEM $(n=4$ independent experiments for each experimental condition).
WT cells, indicating recruitment of $\beta$ arrestin 2, with a delta-BRET of $0.03 \pm 0.002$ (Fig. 8B). Importantly, in GRK6 overexpressing cells, quinpirole induced a significant increase in the delta-BRET $(0.046 \pm$ $0.005, \mathrm{p}<0.05, \mathrm{n}=4$ ) over that in control cells. This effect confirmed that the GRK6-dependent phosphorylation is of importance for the recruitment of $\beta$ arrestin 2 to $D_{2}$ receptors that may have functional consequences for both the $\mathrm{G}$ protein mediated processes and $\beta$ arrestin 2 - mediated signaling ${ }^{6,12}$.

\section{Discussion}

In this study, we investigated the role of GRK6 in behavioral and intracellular signaling manifestations of dopamine deficiency in three different animal models of PD and analyzed responses to acute and chronic L-DOPA in these models. While this study generally highlights the role GRK6 plays in the regulation of dopamine receptors, these observations also indicate that modulation in GRK6 expression or function may provide a basis for enhancement of dopaminergic signaling in conditions when dopamine signaling is limited or correction of abnormal striatal plasticity caused by chronic L-DOPA.

We observed that GRK6-KO mice display a reduction of cataleptic behavior induced by haloperidol compared to wild type controls. A possible mechanism for this effect could be that under conditions of GRK6 deficiency there is an enhanced coupling of striatal $D_{2}$-like receptors to their $G$ proteins ${ }^{12}$. The role of GRK6 in the regulation of $G$ protein-dependent $D_{2} R$ regulation is also indicated by the ability of over-expressed GRK6 to significantly decrease $D_{2} R$ coupling to $G$ proteins ${ }^{12}$ and reduce the effect of $\mathrm{D}_{2} \mathrm{R}$ agonist quinpirole on cAMP levels in cellular systems (Fig. 8). The pronounced $\mathrm{D}_{2}$ Rs supersensitivity in GRK6-KO mice may also favor displacement of antagonist by endogenous agonist, decreasing the ability of haloperidol to induce catalepsy. Furthermore, haloperidol's ability to induce an increase in striatal Akt and GSK3 $\beta$ phosphorylation in WT mice ${ }^{25}$ is significantly blunted in the GRK6-KO mice. It has been demonstrated that the phosphorylation level of Akt is regulated by dopamine through $\beta$ arrestin 2/PP2A pathway leading to Akt dephosphorylation and consequently to a reduction of phosphorylation of its substrate, GSK $3 \beta^{9}$. But when $\mathrm{D}_{2} \mathrm{R}$ is blocked by haloperidol it does not recruit $\beta$ arrestin $2^{33,35}$, and thus causes an increase in levels of pAkt and pGSK3 $\beta$. However, in GRK6-KO mice this effect of haloperidol is significantly reduced. Intriguingly, GRK6-KO mice have also increased basal level of pAkt and pGSK $3 \beta$ compared with the control group. A potential explanation for this phenomenon is that untreated WT mice respond to a basal tone of dopamine action that could maintain the phosphorylation of Akt and GSK3 $\beta$ at reduced level through the $\beta$ arrestin 2 signaling complex, but under conditions of GRK6 deficiency it is likely that the non-phosphorylated or partially phosphorylated $\mathrm{D}_{2} \mathrm{R}$ has reduced ability to engage in $\beta$ arrestin 2-mediated Akt dephosphorylation. In fact, our cell culture experiments directly support this possibility by demonstrating that over-expression of GRK6 increases recruitment of $\beta$ arrestin 2 to $D_{2} R$ following agonist stimulation (Fig. 8). These observations also suggest that $\beta$ arrestin 2-mediated Akt/GSK3 signaling may be generally protective against catalepsy. Intriguingly, a recent study has demonstrated that several $\beta$ arrestin-biased dopamine $\mathrm{D}_{2}$ ligands that are devoid of cataleptic activity in normal mice become cataleptogenic in $\beta$ arrestin 2 knockout mice ${ }^{36}$.

So far, it has been demonstrated that GRK6 is important for the desensitization of $\mathrm{D}_{2} \mathrm{R}^{12,37,38}$; however it remained unclear if striatal GRK6 promoted $\mathrm{D}_{2} \mathrm{R}$ phosphorylation leads also to $\beta$ arrestin 2 recruitment. Since $\beta$ arrestin 2 can play dual roles, both as a terminator of $\mathrm{G}$ protein-mediated signaling (desensitization) and as an inducer of $G$ protein-independent signaling events ${ }^{39}$, it seems likely that GRK6 may be involved in both these processes. Indeed, in other receptor systems, GRK6 is a primary player in regulating $\beta$ arrestin 2 recruitment for $\mathrm{G}$ protein-independent signaling, especially leading 
to Erk activation ${ }^{40-42}$. Here we show that at one dose haloperidol can enhance pAkt level both in WT and GRK6-KO mice. Based on this observation we can hypothesize that GRK6 is not the exclusive kinase involved in $\mathrm{D}_{2} \mathrm{R} / \beta$ arrestin 2 recruitment in the striatum. While the role of GRK6 in the $\mathrm{D}_{2} \mathrm{R}$-mediated AKT/GSK3 signaling cascade still requires further characterization, it is clear that at the behavioral level, an inhibition of GRK6 can potently reverse haloperidol's action on catalepsy.

Hyperdopaminergic mice lacking GRK6 (DAT/GRK6 double KO mice) are more active in comparison to the already-hyperactive DAT-KO mice in a novel environment. When DAT is deficient, the dopamine in the extracellular space cannot be re-captured back into the pre-synaptic terminal, thus causing a prolonged increase in neurotransmitter levels in the extracellular space. It has been shown previously that mice lacking GRK6 are supersensitive to amphetamine and cocaine $\mathrm{e}^{12}$, drugs that induce an increase in dopamine level in the extracellular space. Our present results confirm and extend these observations in a genetic model of hyperdopaminergia. Furthermore, by introduction of GRK6 deficiency to a novel pharmacogenetic model of PD, DDD mice, we demonstrated that LDOPA treatment is more effective in counteracting akinesia under conditions of GRK6 deficiency. It has been shown that DAT-KO mice have reduced levels of striatal $D_{1} R$ and $D_{2} R$, presumably due to continuous DA hyperstimulation ${ }^{26}$, while the opposite was found in mice over-expressing $\mathrm{DAT}^{43}$. In mice lacking both DAT and GRK6, DA receptor sensitivity is likely increased due to lack of GRK6-dependent receptor desensitization as in single GRK6-KO mice as described above, thus providing a stronger response to LDOPA in GRK6-deficient DDD mice.

In the unilateral 6-OHDA lesion hemiparkinsonian model of $\mathrm{PD}$, we observed that the rotational response to chronic L-DOPA was reduced in GRK6-KO mice compared to WT controls. Furthermore, a reduction of L-DOPA induced AIMs was observed in GRK6-KO mice. These results are in contrast to a study by Ahmed et al. ${ }^{15}$, where an increase of L-DOPA induced rotations and AIM score was found under conditions of GRK6 deficiency. However, one should consider the particularities of the unilateral 6-OHDA lesion hemiparkinsonian model of PD: in this model, the rotational bias is determined by the difference in intensity of dopamine signaling between the lesioned and intact striata of animals. In our experimental model, genetic GRK6 deficiency occurs on both sides, so both the intact and lesioned striata have supersensitive dopamine receptors. Thus, both the denervation-related supersensitivity and effects of chronic LDOPA treatment leading to abnormal plasticity in the lesioned side may be less prominent in the GRK6-KO animals that already have supersensitive dopamine receptors on the intact side. Ahmed and colleagues (2010) ${ }^{15}$ used lentivirus to knock-down GRK6 just in the lesioned-side striatum, thus likely creating a greater imbalance in the dopaminergic response between the two sides and exacerbating rotational behavior. While the importance of such dopaminergic imbalance is well established for the induction of rotation ${ }^{44}$, these results also suggest that it is also important for manifestation of dyskinesia in the hemiparkinsonian model of PD. Although at present it is difficult to rule out the possibility of compensatory mechanisms, these observations further indicate an important role of this kinase in dopamine receptor-mediated signaling and desensitization.

It has been reported that dopamine depletion in the striatum induces an increase in the ability of $\mathrm{D}_{1} \mathrm{R}$ to activate $\mathrm{ERK}^{45}$. While chronic treatment with L-DOPA leads to a reduction of ERK activation, L-DOPA treatment is not able to reverse this mechanism in subjects that display dyskinesia ${ }^{13,46}$. The occurrence of sensitized ERK signaling in association with dyskinesia has been described in several studies ${ }^{47,48}$. In agreement with these studies, we found that WT mice that develop significant LIDs have also increased levels of pERK, while GRK6-KO mice that show less pronounced LIDs have also similar pattern of reduced pERK levels relative to WT controls. It has been hypothesized that LIDs are induced by overstimulation of postsynaptic dopamine receptors located on the GABAergic neurons of the dorsal striatum ${ }^{16,49}$. Our model is characterized by GRK6 deficiency that leads to hypersensitivity of dopamine receptors, so logically we should have found an increase of AIMs and so enhanced level of pERK; however, we obtained a reduction for both. While it may reflect altered dopaminergic imbalance in lesioned GRK6-KO mice as described above, it may also indicate the role of GRK6 in regulation of $\mathrm{D}_{1} \mathrm{Rs}$ as recently reported ${ }^{15}$. Furthermore, other signaling pathways in addition to ERK are likely also important for the development of AIMs in mice. A recent study has shown that dopamine depletion and subsequent treatment with L-DOPA enhances activity of the Akt pathway in the striatum ${ }^{22}$. However, in the present study we did not observe significant alterations in Akt and GSK-3 activity between any of the groups tested. These data indicate that the $\mathrm{D}_{2} \mathrm{R}$ triggered Akt/GSK3 pathway may not be critical for the LDOPA-induced rotations and the development of AIMs in 6OHDA mouse model of PD. However, we also did not detect the expected changes in dopamine-dependent Akt and GSK3 activity caused by dopamine denervation 9 or by GRK6 deficiency that is observed in unlesioned GRK6-KO mice (Fig. 2). It should be pointed out that in experiments involving 6-OHDA-lesioned mice all the groups have received chronic L-DOPA treatments, and such pulsatile dopaminergic stimulation may alter the mechanism of regulation of Akt/GSK3 pathway by dopamine.

In conclusion, significant alterations in motor functions observed in animal models of PD that also lack GRK6 suggest the important role of this kinase and its potential involvement in PD manifestations and in responses to antiparkinsonian drugs.

\section{Methods}

Animals. Animal care and treatments were performed in accordance with the Guide for Care and Use of Laboratory Animals (USA National Institutes of Health publication \#865-23, Bethesda, MD) and the protocols were approved by the Italian Ministry of Health. GRK6 and Dopamine Transporter knockout (GRK6-KO and DAT-KO) mice of mixed C57BL/6J x 129Sv/J background were generated as described before ${ }^{12,28}$, and were crossed to generate GRK6/DAT double KO mice. All the single KO and WT littermates were obtained from heterozygous matings, while double $\mathrm{KO}$ mice were obtained from double heterozygous mice. Genotyping was performed in all individuals by PCR (representative PCRs for DAT/GRK6 double KO are shown in Supplementary Fig. S4). Mice of both sexes older than 3 months were used in all these experiments.

Drugs. All the drugs used for this work were purchased from Sigma Aldrich (Milan, Italy) with exception of Carbidopa ((S)-(-)-Carbidopa, Tocris, Bristol, UK) and amphetamine (Poole, UK). All drugs were prepared with a final volume of injection of $10 \mathrm{ml} / \mathrm{kg}$. L-DOPA (3,4-dihydroxyphenylalanine) was freshly dissolved in saline solution and injected i.p. Carbidopa was sonicated first in water containing few microliters of Tween-20 and then diluted in water. Haloperidol was dissolved in few microliters of glacial acetic acid and then diluted in PBS solution. $\alpha$ MT ( $\alpha$-MethylDL-tyrosine methyl ester hydrochloride) and amphetamine were dissolved in saline. Apomorphine (R-(-)-Apomorphine hydrochloride hemihydrates) was dissolved in ascorbate-water $(0.02 \%)$ solution and injected s.c.

Behavioral apparatus and procedure. Catalepsy test. The presence of catalepsy was determined and measured by placing the forepaws of mice on a horizontal wooden bar $(0.7 \mathrm{~cm}$ in diameter), $4 \mathrm{~cm}$ above the tabletop. The time until the mouse removed both forepaws from the bar was recorded, with a maximum cut-off time of 3 minutes. For this test, all the mice were injected either with vehicle as control or with $0.5,1$ or $2 \mathrm{mg} / \mathrm{kg}$ of haloperidol and tested for catalepsy every hour for four hours after treatment.

Locomotor activity test. Locomotor activity was measured in an Omnitech CCDigiscan activity monitor (Accuscan Instruments, Columbus, $\mathrm{OH}$, USA) under bright illumination. To evaluate the impact of mutation or effects of drugs on motor behaviors, mice were placed into activity monitor chambers $(20 \times 20 \mathrm{~cm})$. Various parameters of locomotor activity were measured at 5-min intervals ${ }^{12}$.

Evaluation of acute effects of L-DOPA in DDD mice. To evaluate effects of pharmacologically active compounds under conditions of absolute DA deficiency, the dopamine-deficient DAT-KO mice (DDD mice) model of dopamine deficiency was used $^{26}$. In this experimental procedure we used two groups of mice: DAT-KO and GRK6/DAT double KO mice. Mice were placed to the locomotor chamber for 30 minutes and then were treated with $\alpha$-methyl-p-tyrosine ( $\alpha \mathrm{MT}, 250 \mathrm{mg} / \mathrm{kg}$, i.p.), a 
potent irreversible inhibitor of Tyrosine Hydroxylase (TH), to eliminate dopamine in the brain ${ }^{26}$. One hour after $\alpha \mathrm{MT}$, the animals, received an injection of L-DOPA/ Carbidopa (10/10 mg/kg, i.p.), followed by another dose of only L-DOPA $(20 \mathrm{mg} / \mathrm{kg}$, i.p.) one hour later. In a second group of animals, one hour after $\alpha \mathrm{MT}$ injection the animals received an injection of L-DOPA/Carbidopa at 30/10 mg/kg (i.p.).

Evaluation of chronic effects of L-DOPA in unilaterally 6-OHDA lesioned mice. 6-Hydroxy-Dopamine (6-OHDA) striatal lesion in mice. Dopamine neuron lesions were performed by unilateral injection of 6-OHDA into the striatum. 30 minutes before the neurotoxin injection all the animals received a desipramine $35 \mathrm{mg} / \mathrm{kg}$ injection (i.p., dissolved in saline) to protect the noradrenergic innervations into the striatum. Then the animals were anesthetized by a mixture of isoflurane/oxygen and placed on a stereotaxic apparatus (David Kopf instrument, Tujunga, CA, USA) with mouse adaptor and lateral ear bars. The skin on the skull was cut and two holes were made on the same side by a surgical drill. The coordinates were chosen in according to Franklin and Paxinos atlas $(1997)^{50}: \mathrm{AP}=+1, \mathrm{~L}=-2.1, \mathrm{DV}=-3$ and $\mathrm{AP}=+0.4$, $\mathrm{L}=-2.3, \mathrm{DV}=-3$. The injections were performed by using injection cannulae $(31$ Gauge) connected with a polyethylene tube to a $1 \mathrm{ml}$ Hamilton Syringe at the $0.7 \mu \mathrm{l} /$ min rate; to let the solution diffuse in the tissue the cannulae was kept in the same position 3 minutes more. 6-OHDA was dissolved in $0.02 \%$ ascorbate - artificial CSF (CMA/Microdialysis, Solna, Sweden) solution at the concentration of $6 \mu \mathrm{g} / \mu \mathrm{l} .2 \mu \mathrm{l}$ of this solution was used for infusion into striatum in both injections. In order to assess the lesion grade, at the end of the behavioral studies the striatum were collected and the $\mathrm{TH}$ expression was evaluated by Western blot analysis.

Circling Behavior. To measure circling behavior we used glass cylinders with a diameter of $19 \mathrm{~cm}$ and height of $25 \mathrm{~cm}$. All animals were recorded by video camera for 1 hour immediately after treatment and were scored for rotations. Each $360^{\circ}$ rotation of the body axes was counted as a rotation.

Abnormal Involuntary Movements (AIMs). 6-OHDA-lesioned mice were monitored for abnormal involuntary movements for one minute every 20 minutes during the rotational screening after L-DOPA treatment ${ }^{31}$ with minor modification. AIMs were divided into two subtypes, axial and limb AIMs. Each of these subtypes was scored on a severity scale from 0 to $4(0=$ no dyskinesia; $1=$ occasional dyskinesia displayed less than $50 \%$ of the observation time; $2=$ sustained dyskinesia displayed more than $50 \%$ of observation time; $3=$ continous dyskinesia; $4=$ continous dyskinesia not interruptible by outer stimuli). The level of dyskinesia was counted as a sum of each score in the different time points. In this study, orolingual $\mathrm{AIMs}^{31}$ were not analyzed because of difficulties in assessing this parameter in our experimental conditions.

The experimental procedure for chronic L-DOPA treatment. Twenty eight days after the surgery, 6-OHDA-lesioned and sham-operated WT and GRK6-KO mice were treated with L-DOPA/Carbidopa $(20 / 12 \mathrm{mg} / \mathrm{kg}$, i.p.) for 21 days and they were tested every week for circling behavior and AIMs immediately after the injections).

Antibodies and Western Blot Analyses. The anti-phospho Akt (Thr-308), anti-Akt, anti-phosphoGSK3 $\beta$ (Ser-9), anti-GSK3 $\beta$, anti-phosphoERK1/2 and anti-ERK1/2 were purchased from Cell Signaling Technology (Beverly, MA); while the antiTyrosine Hydroxylase antibody was purchased from Santa Cruz Biotechnology (Heidelberg, Germany) and the anti-actin antibody from Sigma Aldrich (Milan, Italy). Western blot analyses of brain samples were performed as described ${ }^{9}$. Briefly, mice were euthanized by decapitation, after which the heads of the animals were immediately cooled by immersion in liquid nitrogen for $6 \mathrm{~s}$. Both hemispheres of striatum were rapidly dissected (within $60 \mathrm{~s}$ ) on an ice-cold surface and frozen in liquid nitrogen before protein extraction. Tissue samples were homogenized in boiling $1 \%$ SDS solution supplemented with inhibitor of protease (Hoffmann-LaRoche, Basel, Switzerland) and phosphatase (Termo Scientific, MA, USA) and boiled for $10 \mathrm{~min}$. Protein concentrations were measured using a DC-protein assay (BioRad, Hercules, CA). Protein extracts $(25 \mu \mathrm{g})$ were separated on 10\% SDS/PAGE and transferred to nitrocellulose membranes. Blots were incubated with primary antibodies overnight at $4{ }^{\circ} \mathrm{C}$. Immune complexes were detected using appropriate peroxidase-conjugated secondary antibodies (Jackson Immuno-Research, West Grove, PA) and a chemiluminescent reagent (SuperSignal West-Pico; Pierce Biotechnology, Rockford, IL). Densitometric analysis was performed by IMAGEJ software. For quantitative analysis, total proteins were used as loading controls for phosphoprotein signals. Results were normalized to respective control conditions and presented as means \pm SEM.

BRET assay to analyze cAMP regulation and $\beta$ arrestin 2 recruitment to D2 dopamine receptor following GRK6 over-expression in vitro. The experiments were performed in Human embryonic kidney 293 cells (HEK293T) cells with overexpressed GRK6 as described ${ }^{12,33,34}$ (see Supplementary Information for details).

Statistical analysis. The catalepsy data for each dose of haloperidol were analyzed by Two-Way ANOVA repeated measure with Genotype (WT and GRK6-KO) and Treatment (Saline and Haloperidol) as between factor and Time (four time point) as repeated measures factor. The basal locomotor activity of DAT-KO and DAT/GRK6KO was analyzed by One-Way ANOVA for repeated measures with Genotype (DATKO and DAT/GRK6-KO) as between factor and Time (24 time points) as repeated measures. The effect of L-DOPA in DDD mice was analyzed by One-Way ANOVA for repeated measures with Genotype (DAT-KO and DAT/GRK6-KO) as between factor and time (37 time points) as repeated measures. The effect of L-DOPA chronic treatment on the hemiparkinsonian model were analyzed by Two-Way ANOVA with Genotype (WT and GRK6-KO) and Lesion (sham-operated and lesioned) as between factor and Time (Day1, Day7, Day14, Day21) as repeated measures. The AIMs score were analyzed by One-Way ANOVA for repeated measures with Genotype (WT and GRK6-KO) as between factor and Time (Day1, Day7, Day14, Day21) as repeated measures. Neurochemical data for the dose response of haloperidol in the catalepsy experiment were analyzed by One-Way ANOVA with Treatment (Saline, 1 and $2 \mathrm{mg} / \mathrm{kg}$ of haloperidol) as between factor. Tukey honestly significant difference (HSD) or Bonferroni post hoc analyses were used when appropriate. The other neurochemical and biochemical data were analyzed by either non-parametric oneway ANOVA or Mann-Whitney u-test.

1. Fearnley, J. M. \& Lees, A. J. Ageing and Parkinson's disease: substantia nigra regional selectivity. Brain 114 (Pt5), 2283-2301 (1991).

2. Kish, S. J., Shannak, K. \& Hornykiewicz, O. Uneven pattern of dopamine loss in the striatum of patients with idiopathic Parkinson's disease. Pathophysiologic and clinical implications. N Engl J Med 318, 876-880 (1988).

3. Bezard, E., Brotchie, J. M. \& Gross, C. E. Pathophysiology of levodopa-induced dyskinesia: potential for new therapies. Nat Rev Neurosci 2, 577-588 (2001).

4. Cotzias, G. C., Papavasiliou, P. S. \& Gellene, R. Modification of Parkinsonism-chronic treatment with L-dopa. N Engl J Med 280, 337-345 (1969).

5. Stocchi, F., Nordera, G. \& Marsden, C. D. Strategies for treating patients with advanced Parkinson's disease with disastrous fluctuations and dyskinesias. Clin Neuropharmacol 20, 95-115 (1997).

6. Beaulieu, J. M. \& Gainetdinov, R. R. The physiology, signaling, and pharmacology of dopamine receptors. Pharmacol Rev 63, 182-217 (2011).

7. Missale, C., Nash, S. R., Robinson, S. W., Jaber, M. \& Caron, M. G. Dopamine receptors: from structure to function. Physiol Rev 78, 189-225 (1998).

8. Ferguson, S. S. Evolving concepts in G protein-coupled receptor endocytosis: the role in receptor desensitization and signaling. Pharmacol Rev 53, 1-24 (2001).

9. Beaulieu, J. M. et al. Lithium antagonizes dopamine-dependent behaviors mediated by an AKT/glycogen synthase kinase 3 signaling cascade. Proc Natl Acad Sci U S A 101, 5099-5104 (2004).

10. Lefkowitz, R. J. \& Shenoy, S. K. Transduction of receptor signals by beta-arrestins. Science 308, 512-517 (2005).

11. Erdtmann-Vourliotis, M., Mayer, P., Ammon, S., Riechert, U. \& Hollt, V. Distribution of G-protein-coupled receptor kinase (GRK) isoforms 2, 3, 5 and 6 mRNA in the rat brain. Brain Res Mol Brain Res 95, 129-137 (2001).

12. Gainetdinov, R. R. et al. Dopaminergic supersensitivity in G protein-coupled receptor kinase 6-deficient mice. Neuron 38, 291-303 (2003)

13. Bezard, E. et al. L-DOPA reverses the MPTP-induced elevation of the arrestin2 and GRK6 expression and enhanced ERK activation in monkey brain. Neurobiol Dis 18, 323-335 (2005).

14. Ahmed, M. R., Bychkov, E., Gurevich, V. V., Benovic, J. L. \& Gurevich, E. V. Altered expression and subcellular distribution of GRK subtypes in the dopamine-depleted rat basal ganglia is not normalized by l-DOPA treatment. J Neurochem 104, 1622-1636 (2008).

15. Ahmed, M. R. et al. Lentiviral overexpression of GRK6 alleviates L-dopa-induced dyskinesia in experimental Parkinson's disease. Sci Transl Med 2, 28ra28 (2010).

16. Aubert, I. et al. Increased D1 dopamine receptor signaling in levodopa-induced dyskinesia. Ann Neurol 57, 17-26 (2005).

17. Cenci, M. A., Lee, C. S. \& Bjorklund, A. L-DOPA-induced dyskinesia in the rat is associated with striatal overexpression of prodynorphin- and glutamic acid decarboxylase mRNA. Eur J Neurosci 10, 2694-2706 (1998).

18. Sgambato-Faure, V. et al. Coordinated and spatial upregulation of arc in striatonigral neurons correlates with L-dopa-induced behavioral sensitization in dyskinetic rats. J Neuropathol Exp Neurol 64, 936-947 (2005).

19. Gold, S. J. et al. RGS9-2 negatively modulates L-3, 4-dihydroxyphenylalanineinduced dyskinesia in experimental Parkinson's disease. J Neurosci 27, 1433814348 (2007)

20. Bezard, E. et al. Attenuation of levodopa-induced dyskinesia by normalizing dopamine D3 receptor function. Nat Med 9, 762-767 (2003).

21. Bordet, R. et al. Induction of dopamine D3 receptor expression as a mechanism of behavioral sensitization to levodopa. Proc Natl Acad Sci U S A 94, 3363-3367 (1997).

22. Bychkov, E., Ahmed, M. R., Dalby, K. N. \& Gurevich, E. V. Dopamine depletion and subsequent treatment with L-DOPA, but not the long-lived dopamine agonist pergolide, enhances activity of the Akt pathway in the rat striatum. J Neurochem 102, 699-711 (2007).

23. Boulay, D. et al. Haloperidol-induced catalepsy is absent in dopamine $\mathrm{D}(2)$, but maintained in dopamine D(3) receptor knock-out mice. Eur J Pharmacol 391, 6373 (2000).

24. Beaulieu, J. M. et al. An Akt/beta-arrestin 2/PP2A signaling complex mediates dopaminergic neurotransmission and behavior. Cell 122, 261-273 (2005).

25. Emamian, E. S., Hall, D., Birnbaum, M. J., Karayiorgou, M. \& Gogos, J. A. Convergent evidence for impaired AKT1-GSK3beta signaling in schizophrenia. Nat Genet 36, 131-137 (2004). 
26. Sotnikova, T. D. et al. Dopamine-independent locomotor actions of amphetamines in a novel acute mouse model of Parkinson disease. PLoS Biol 3, e271 (2005).

27. Gainetdinov, R. R. \& Caron, M. G. Monoamine transporters: from genes to behavior. Annu Rev Pharmacol Toxicol 43, 261-284 (2003).

28. Giros, B., Jaber, M., Jones, S. R., Wightman, R. M. \& Caron, M. G. Hyperlocomotion and indifference to cocaine and amphetamine in mice lacking the dopamine transporter. Nature 379, 606-612 (1996).

29. Costa, R. M. et al. Rapid alterations in corticostriatal ensemble coordination during acute dopamine-dependent motor dysfunction. Neuron 52, 359-369 (2006).

30. Lundblad, M., Picconi, B., Lindgren, H. \& Cenci, M. A. A model of L-DOPAinduced dyskinesia in 6-hydroxydopamine lesioned mice: relation to motor and cellular parameters of nigrostriatal function. Neurobiol Dis 16, 110-123 (2004).

31. Winkler, C., Kirik, D., Bjorklund, A. \& Cenci, M. A. L-DOPA-induced dyskinesia in the intrastriatal 6-hydroxydopamine model of parkinson's disease: relation to motor and cellular parameters of nigrostriatal function. Neurobiol Dis 10, 165186 (2002).

32. Santini, E. et al. Critical involvement of cAMP/DARPP-32 and extracellular signal-regulated protein kinase signaling in L-DOPA-induced dyskinesia. J Neurosci 27, 6995-7005 (2007).

33. Masri, B. et al. Antagonism of dopamine D2 receptor/beta-arrestin 2 interaction is a common property of clinically effective antipsychotics. Proc Natl Acad Sci U S A 105, 13656-13661 (2008)

34. Barak, L. S. et al. Pharmacological characterization of membrane-expressed human trace amine-associated receptor 1 (TAAR1) by a bioluminescence resonance energy transfer cAMP biosensor. Mol Pharmacol 74, 585-594 (2008).

35. Klewe, I. V. et al. Recruitment of beta-arrestin2 to the dopamine D2 receptor: insights into anti-psychotic and anti-parkinsonian drug receptor signaling. Neuropharmacology 54, 1215-1222 (2008).

36. Allen, J. A. et al. Discovery of $\{$ beta\}-Arrestin-Biased Dopamine D2 Ligands for Probing Signal Transduction Pathways Essential for Antipsychotic Efficacy. Proc Natl Acad Sci U S A 108, 18488-18493 (2011).

37. Gainetdinov, R. R., Premont, R. T., Bohn, L. M., Lefkowitz, R. J. \& Caron, M. G. Desensitization of $\mathrm{G}$ protein-coupled receptors and neuronal functions. Annu Rev Neurosci 27, 107-144 (2004).

38. Premont, R. T. \& Gainetdinov, R. R. Physiological roles of G protein-coupled receptor kinases and arrestins. Annu Rev Physiol 69, 511-534 (2007).

39. Luttrell, L. M. \& Lefkowitz, R. J. The role of beta-arrestins in the termination and transduction of G-protein-coupled receptor signals. J Cell Sci 115, 455-465 (2002)

40. Kim, J. et al. Functional antagonism of different G protein-coupled receptor kinases for beta-arrestin-mediated angiotensin II receptor signaling. Proc Natl Acad Sci U S A 102, 1442-1447 (2005).

41. Rajagopal, K. et al. Beta-arrestin2-mediated inotropic effects of the angiotensin II type 1A receptor in isolated cardiac myocytes. Proc Natl Acad Sci U S A 103 16284-16289 (2006).

42. Ren, X. R. et al. Different G protein-coupled receptor kinases govern G protein and beta-arrestin-mediated signaling of V2 vasopressin receptor. Proc Natl Acad Sci U S A 102, 1448-1453 (2005).
43. Ghisi, V. et al. Reduced D2-mediated signaling activity and trans-synaptic upregulation of D1 and D2 dopamine receptors in mice overexpressing the dopamine transporter. Cell Signal 21, 87-94 (2009).

44. Ungerstedt, U. \& Arbuthnott, G. W. Quantitative recording of rotational behavior in rats after 6-hydroxy-dopamine lesions of the nigrostriatal dopamine system. Brain Res 24, 485-493 (1970).

45. Gerfen, C. R., Miyachi, S., Paletzki, R. \& Brown, P. D1 dopamine receptor supersensitivity in the dopamine-depleted striatum results from a switch in the regulation of ERK1/2/MAP kinase. J Neurosci 22, 5042-5054 (2002).

46. Kim, D. S., Palmiter, R. D., Cummins, A. \& Gerfen, C. R. Reversal of supersensitive striatal dopamine D1 receptor signaling and extracellular signal-regulated kinase activity in dopamine-deficient mice. Neuroscience 137, 1381-1388 (2006).

47. Pavon, N., Martin, A. B., Mendialdua, A. \& Moratalla, R. ERK phosphorylation and FosB expression are associated with L-DOPA-induced dyskinesia in hemiparkinsonian mice. Biol Psychiatry 59, 64-74 (2006).

48. Santini, E. et al. L-DOPA activates ERK signaling and phosphorylates histone H3 in the striatonigral medium spiny neurons of hemiparkinsonian mice. J Neurochem 108, 621-633 (2009).

49. Santini, E., Valjent, E. \& Fisone, G. Parkinson's disease: levodopa-induced dyskinesia and signal transduction. FEBS J 275, 1392-1399 (2008).

50. Franklin, K. B. J. \& Paxinos, G. The mouse brain in stereotaxic coordinates. (Academic Press, 1997).

51. Cenci, M. A. \& Lundblad, M. Ratings of L-DOPA-induced dyskinesia in the unilateral 6-OHDA lesion model of Parkinson's disease in rats and mice. Curr Protoc Neurosci Chapter 9, Unit 925 (2007).

\section{Acknowledgements:}

Supported in part by research awards to Raul R. Gainetdinov from F. Hoffmann - La Roche Ltd. (Basel, Swtitzerland) and Fondazione Compagnia di San Paolo (Torino, Italy) and to RTP (NIH DA016347)

\section{Author contributions}

F.M., S.E., A.S., T.D.S., M.G.C., R.T.P. and R.R.G. conceived and designed the experiments F.M., S.E., T.D.S. and R.R.G. executed the experiments and analyzed the data. All authors wrote and reviewed the manuscript.

\section{Additional information}

Supplementary information accompanies this paper at http://www.nature.com/ scientificreports

Competing financial interests: The authors declare no competing financial interests. License: This work is licensed under a Creative Commons Attribution-NonCommercial-ShareAlike 3.0 Unported License. To view a copy of this license, visit http://creativecommons.org/licenses/by-nc-sa/3.0/

How to cite this article: Managò, F. et al. The role of GRK6 in animal models of Parkinson's Disease and L-DOPA treatment. Sci. Rep. 2, 301; DOI:10.1038/srep00301 (2012). 